\title{
A diversidade inventarial de Coleoptera (Insecta) em uma paisagem antropizada do Bioma Araucária ${ }^{1}$
}

\author{
Norma G. Ganho ${ }^{2,4} \&$ Renato C. Marinoni ${ }^{3,4}$
}

${ }^{1}$ Contribuição número 1544 do Departamento de Zoologia, Universidade Federal do Paraná.

${ }^{2}$ Centro de Ciências Agrárias e Ambientais, Pontifícia Universidade Católica do Paraná, Caixa Postal 129, 83010-500 São José dos Pinhais-PR, Brasil.jomario@uol.com.br

${ }^{3}$ Departamento de Zoologia, Universidade Federal do Paraná, Caixa Postal 19020, 81531-980 Curitiba-PR, Brasil. rcmari@ufpr.br ${ }^{4}$ Bolsistas do CNPq.

\begin{abstract}
The inventory diversity of Coleoptera (Insecta) of an anthropized landscape in the Biome Araucaria. The species richness, taxonomic composition, rare species, and taxonomic constancy at family level were studied in communities of Coleoptera in Vila Velha, Ponta Grossa, Paraná, as part of PROVIVE project. The data were gathered through malaise traps in the understory stratum of five sites with different floristic conditions product of anthropogenic effects, from September/1999 to August/2000. The 52 weeks sampling in the five sites produced 10,822 specimens of 1,659 species. All areas present high species richness and high diversity as indicated by various indices. The area in advanced condition of vegetation succession was less rich than ones in early/middle stage. According to different species richness estimators, the number of observed species could be increased by $22-123 \%$ if more time colleting effort had been made. The early successional stage areas were richer in rare species (singletons, doubletons and uniques) than the more preserved ones. In the five areas there were a taxonomic constancy among the richest families (Curculionidae, Chrysomelidae, Cerambycidae, Staphylinidae, Mordelidae, Elateridae, Scarabaeidae, Coccinellidae and Tenebrionidae) involving $60 \%$ of the total species as observed with the specimens abundance. If the existence of this pattern is confirmed, it will be easier and faster to study Coleoptera communities and soon apply this order as a forest environmental indicator taxon.
\end{abstract}

KEYWORDS. Alpha and gamma diversity; Araucaria forest; bioindicator; family and species richness; PROVIVE.

RESUMO. A diversidade inventarial de Coleoptera (Insecta) em uma paisagem antropizada do Bioma Araucária. Como parte do Projeto PROVIVE, foram analisadas a riqueza de espécies, a composição taxonômica, a proporção de espécies raras e a constância taxonômica ao nível de família relacionada à riqueza de espécies, em comunidades de Coleoptera, em Vila Velha, Ponta Grossa, Paraná. Os dados foram obtidos a partir de coletas através de armadilha malaise no estrato do sub-bosque de cinco áreas com diferentes graus de intervenção antrópica, de setembro de 1999 a agosto de 2000. As 52 semanas de amostragem nas cinco áreas resultaram na coleta de 10.822 indivíduos de 1659 espécies. Todas as áreas apresentaram alta riqueza de espécies e diversidade, como indicado por vários índices. A área em estágio mais avançado de sucessão vegetal foi menos rica do que aquelas em estágio inicial/intermediária. De acordo com diferentes estimadores de riqueza de espécies, o número de espécies coletadas poderia aumentar de 22-123\% com o aumento do esforço de coleta. As áreas menos conservadas foram mais ricas em espécies raras ("singletons", "doubletons" e únicas) do que as mais conservadas. Nas cinco áreas houve uma constância taxonômica entre as famílias mais ricas (Curculionidae, Chrysomelidae, Cerambycidae, Staphylinidae, Mordelidae, Elateridae, Scarabaeidae, Coccinellidae e Tenebrionidae) envolvendo $60 \%$ do total de espécies, como observado para a abundância de indivíduos. A existência de um padrão de constância taxonômica de famílias, quando considerados $60 \%$ da riqueza de espécies e/ou de abundância de indivíduos por local, poderá tornar mais fácil e rápido o estudo de comunidades de Coleoptera, habilitando a ordem a ser um táxon indicador de condições ambientais de áreas florestadas.

PALAVRAS-CHAVE. Bioindicador; diversidade alfa e gama; floresta de araucária; PROVIVE; riqueza de espécies e famílias.

A riqueza de espécies anotada em um inventário e a comparação entre as riquezas de espécies observadas em diferentes inventários vêm sendo discutidas mais profundamente desde Whittaker (1960; 1972). Assim, foi reconhecida a existência de uma diversidade de espécies inventarial e de uma diversidade de espécies diferencial (Whittaker 1960, 1972; Magurran 2004). A primeira trata da riqueza de espécies (e abundância) em diferentes níveis, desde a diversidade pontual, refletindo a riqueza de espécies produto de uma amostra, passando pela diversidade $\alpha$ (alfa) dentro de um habitat e pela diversidade $\gamma$ (gama) (soma das espécies dentro de uma paisagem), até a diversidade $\varepsilon$ (épsilon), resultado da soma de todas as espécies de um táxon dentro de uma província biogeográfica. Por outro lado, a diversidade diferencial analisa as diferenças existentes entre os vários níveis da diversidade inventarial, desde entre amostras dentro de um habitat, passando pelas comparações entre habitats dentro de uma paisagem (diversidade $\beta$, beta), até a diversidade entre paisagens dentro de uma província biogeográfica (diversidade $\delta$, delta) (Harrison et al. 1992; Moreno \& Halffter 2001; Koleff et al. 2003). Apesar de não haver dúvida quanto ao objeto do resultado numérico de cada um destes conceitos, ou seja, a diversidade inventarial reflete a soma das espécies (índices de diversidade introduzem também o atributo abundância nos 
cálculos), e a diversidade diferencial trata de analisar a diferença que se observa entre as riquezas de espécies, há certo questionamento quanto à abrangência espacial ou temporal que se deva dar a cada um dos tipos de diversidade.

Considerando que em todos os ambientes, mesmo nos mais homogêneos, há uma modificação contínua dos habitats como resultado da interação de diferentes elementos abióticos e bióticos, as comunidades animais também são afetadas em sua composição e estrutura. As diferenças em composição e estrutura das comunidades sendo mais substanciais quanto menor for a mobilidade dos grupos animais e maior a filopatria. Desta forma, a conceituação dos diferentes níveis de diversidade diferencial não será uma questão apenas de escala física espacial/temporal, mas também de comportamento do táxon animal sob análise. No caso dos Insecta, alguns fatores avultam, como: 1) o ciclo biológico, principalmente das espécies holometábolas (muitas com fases de vida em diferentes habitats), com a grande maioria das espécies apresentando ciclo de vida anual, e algumas até bivoltismo e multivoltismo; 2) a sazonalidade decorrente do ciclo de vida associado a fatores climáticos e à disponibilidade trófica (principalmente dos herbívoros); 3) e, ainda, as características locomotoras (voadoras ou ambulatórias) que têm implicações na metodologia de coleta a ser empregada.

No Parque Estadual de Vila Velha, em Ponta Grossa, Paraná, numa paisagem caracterizada como um capão de araucárias, foi possível reconhecer a existência de cinco áreas alteradas de diferentes formas pela ação humana. O conhecimento do histórico desta ação antrópica e as diferentes condições florísticas (composição e estrutura) de cada uma das áreas permitiram estabelecer um protocolo de coleta de Insecta, admitindo-se que estas áreas representavam diferentes habitats de uma paisagem (capão de araucária de uma Floresta Ombrófila Mista). Com apoio nos conceitos de diversidade inventarial e de diversidade diferencial (acima expostos) e no conceito de paisagem, conforme proposto por Metzger (2001), optou-se por considerar que, apesar da coleta ter sido por armadilha malaise (diversidade pontual), tratar-se-iam de estudos de diversidade inventarial alfa (dentro de um habitat) e de diversidade diferencial beta (entre habitats) dentro de uma paisagem, com o número total de espécies refletindo a diversidade inventarial gama (paisagem).

Vários trabalhos relacionaram a fauna de Coleoptera ao ambiente florestal, enfocando a importância da diversidade, estrutura e arquitetura vegetais (Southwood et al. 1979; Lawton 1983; Lawton et al. 1998; Humphrey et al. 1999; Wagner 2000), na diversidade deste grupo de insetos. Em decorrência destas características, vários estudos (Hutcheson 1990, Marinoni \& Dutra 1997, Humphrey et al. 1999, Hutcheson \& Jones 1999) têm encontrado elementos que apontam os Coleoptera como possíveis indicadores de diversidade e de ambiente, principalmente na determinação de níveis de conservação de áreas florestadas.

Estudos anteriores apoiados no inventário realizado no Parque Estadual de Vila Velha, em que foram feitas análises da Ordem Coleoptera, através de dados de riqueza de famílias e suas abundâncias, tanto do material capturado através de armadilha malaise quanto através de armadilha de solo, já indicavam que a comunidade de Coleoptera se apresentava diferenciada entre as áreas. Estas diferenças corroborando os diferentes níveis de perturbação antrópica, e caracterizavam a existência de diferentes habitats (Ganho \& Marinoni 2003; Marinoni \& Ganho 2003).

O presente estudo, realizado no Parque Estadual de Vila Velha, Ponta Grossa, dentro do projeto PROVIVE, visou avaliar a riqueza e a abundância de espécies de Coleoptera, coletadas por armadilha malaise, e a existência de diferentes características na composição e na estrutura das comunidades de Coleoptera, relacionando-as às diferentes condições de manejo e de conservação das cinco áreas. Os seguintes itens foram verificados: 1) a variabilidade na riqueza de espécies das áreas como decorrência das características diferenciadas da vegetação; 2) o potencial das áreas para apresentarem uma maior riqueza de espécies, além daquela efetivamente observada, através de cálculos de estimativas; 3 ) o período de tempo no qual, através do mesmo esforço de coleta, a curva de acumulação de espécies se aproxima da assíntota; 4) a possível existência de padrões relativos à presença de espécies comuns e raras nas áreas com diferentes níveis de ação antrópica; 5) se ocorre uma constância taxonômica entre as famílias com maior riqueza de espécies para servir como potencial indicador ecológico.

\section{MATERIAL E MÉTODOS}

O inventário foi realizado, entre 30 de agosto de 1999 a 28 de agosto de 2000, no Parque Estadual de Vila Velha, localizado no município de Ponta Grossa, Estado do Paraná, junto à Rodovia do Café, BR 376, Km 83.

Características da região. O Parque Estadual de Vila Velha é uma área de preservação do Estado do Paraná, com área de $5.032 .384,00 \mathrm{~m}^{2}$, e altitude média de $880 \mathrm{~m}$, onde predomina Floresta Ombrófila Mista Montana (Veloso \& Góes-Filho 1982, adotada pelo IBGE 1992), com diferentes níveis de interferência antrópica.

O clima, pela classificação de Koeppen, é temperado sempre úmido, caracterizado por apresentar estações do ano bem definidas, sendo os invernos frios, com mais de cinco geadas noturnas por ano; verões quentes e chuvas bem distribuídas ao longo do ano; outros fatores importantes são a variação da intensidade de irradiação solar e do fotoperíodo no decorrer do ano, que conferem um caráter temperado ao clima (Maack 1981).

A vegetação da região foi definida por Maack (op.cit.) como de campos limpos com capões de matas de araucária, onde também se destacam coníferas e lauráceas em múltiplas associações e agrupamentos, com matas ciliares ao longo dos rios e arroios.

Foram inventariadas cinco áreas em diferentes estágios de sucessão vegetal ou em diferentes condições ambientais em função de ações de manejo. Estas áreas têm as características mais importantes descritas abaixo, já a situação no espaço e a 
descrição detalhada foram apresentadas em Ganho \& Marinoni (2003).

Área de borda $\left(25^{\circ} 13^{\prime} 5^{\prime \prime} \mathrm{S}, 50^{\circ} 2^{\prime} 26,9^{\prime \prime} \mathrm{W}\right)$. Caracterizada como de transição entre vegetação arbórea em estágio intermediário de sucessão e área de campo, mantida por roçadura (pelo menos semestralmente), exceto por uma faixa de 10 metros de raio em torno da armadilha.

Área de araucária $\left(25^{\circ} 13^{\prime} 5,9^{\prime \prime} \mathrm{S}, 50^{\circ} 2^{\prime} 31,2^{\prime \prime} \mathrm{W}\right)$. Armadilha situada a aproximadamente 240 metros da área de borda, em um povoamento florestal de Araucaria angustifolia (pinheirodo-paraná), onde esta espécie é dominante em biomassa e cujo manejo de limpeza deixou de ser feito por volta de 1981. Os pinheiros têm aproximadamente 25 metros de altura e o estrato médio alcança de 10 a 15 metros.

Área fase 1 ( $2513^{\prime} 13,3^{\prime \prime} \mathrm{S}, 50^{\circ} 2^{\prime}$ 14,1" W). Armadilha situada a 335 metros da área de borda, em área de aproximadamente 15 hectares, anteriormente utilizada para culturas agrícolas sazonais, como milho e feijão, e em processo de regeneração natural desde 1984. Encontra-se numa fase inicial a intermediária de sucessão vegetal. Apresenta um dossel bastante aberto, que permite uma passagem intensa de luz; no estrato superior visualizam-se árvores de grande porte ( 20 a 25 metros de altura).

Área fase $2\left(25^{\circ} 13^{\prime} 2,9^{\prime \prime} \mathrm{S}, 50^{\circ} 2^{\prime} 14,1^{\prime \prime} \mathrm{W}\right)$. Armadilha situada a 400 metros da área de borda, aproximadamente. Nesta área foram realizadas as coletas do "Projeto de Levantamento da Fauna Entomológica no Estado do Paraná" (PROFAUPAR) de agosto de 1986 a julho de 1988. Floresta primária alterada pela retirada de várias essências vegetais, como pinheiro-doparaná, imbuia, canelas diversas e algumas mirtáceas. A sucessão vegetal varia de intermediária a avançada, dependendo do ponto observado. É pobre em epífitas e possui o dossel fechado com copas atingindo entre 20 e 25 metros.

Área fase $3\left(25^{\circ} 13^{\prime} 27,6^{\prime \prime} \mathrm{S}, 50^{\circ} 1^{\prime} 52,7^{\prime \prime} \mathrm{W}\right)$. Armadilha situada a 1200 metros da área denominada de borda e a 90 metros da borda da floresta, aproximadamente. Floresta primária alterada por cortes seletivos. É a mais bem conservada dentre todas, com estrutura definida e homogênea. As características da flora são muito semelhantes às da área fase 2, porém com uma maior densidade de araucária, epífitas e lianas. Além disso, as imbuias apresentam-se maiores (altura e massa), bem como as demais lauráceas (canelas), atingindo aproximadamente 30 metros de altura.

Método de coleta. Foi utilizada uma armadilha malaise (Townes 1972) em cada uma das áreas. Esta armadilha é seletiva na medida que captura insetos que voam no estrato florestal, correspondente a uma parte do sub-bosque, que vai do solo à altura de aproximadamente 1,00 a 1,20 m. O material foi retirado do frasco coletor, semanalmente, durante um ano.

Material e identificação. Os besouros foram reconhecidos nominalmente ao nível de família, e dentro destas como morfoespécies. Informações sobre a identificação do material entomológico podem ser encontradas em Ganho \& Marinoni (2003). Para facilitar o acesso às informações, os exemplares coletados foram registrados em um banco de dados relacional gerenciado pelo programa MS Access 2000, desenvolvido por
S.R. Bonatto (Universidade Federal do Paraná). O material coletado está depositado na Coleção de Entomologia Pe. Jesus Santiago Moure, no Departamento de Zoologia da Universidade Federal do Paraná.

Análises de dados. Ao lado dos diferentes índices ecológicos (Magurran 1988, 2004), os estimadores nãoparamétricos de riqueza de espécies (Colwell \& Coddington 1994) têm propiciado a comparação da diversidade entre diferentes áreas. Há várias críticas negativas ao emprego dos índices de diversidade de espécies para comparações entre ecossistemas, tendo em vista as variações que decorrem das influências nos dados amostrais, dentre outras: de faunas local e regional que apresentam valores de diversidade semelhantes apesar de serem muito diferentes na composição de espécies (Penev 1996; Scott \& Anderson 2003); dos diferentes métodos de coleta, do tempo e período dos levantamentos, e ainda pelas diferentes maneiras com que se ponderam os dados de abundância e de riqueza de espécies (Bullock 1971; Alatalo \& Alatalo 1977; Samways 1995). Como se referiu Bullock (1971), os índices não mediriam um caráter intrínseco da comunidade, mas seriam a expressão da diversidade do habitat em termos do seu efeito sobre a fauna. Neste sentido, discutimos os valores de alguns índices neste trabalho. Da mesma forma, os estimadores de riqueza de espécies têm sido utilizados sem que haja uma definição de qual é aquele que melhor indica a riqueza de uma área (Palmer 1990; Coddington et al. 1996; Gotelli \& Colwell 2001). Mas, os vários trabalhos que apresentaram estimativas utilizando diferentes cálculos nãoparamétricos têm mostrado que um ordenamento hierárquico dos valores estimados de riqueza de espécies aponta para uma classificação semelhante dos métodos. Ou seja, os métodos que estimam as mais altas riquezas são quase sempre os mesmos, assim como aqueles métodos que estimam as mais baixas riquezas. O emprego dos vários estimadores em contínuos inventários em uma mesma área deverá contribuir para indicar qual deles é o melhor.

Para o cálculo do índice de Brillouin, os valores de $\boldsymbol{n}$ fatorial foram obtidos através do método proposto por Oliveira et al. (1998). Os demais índices ecológicos foram obtidos através do programa Bio Diversity Professional Beta (McAleece 1998). Os cálculos de estimativa de riqueza pelos métodos Chao 1, Chao 2, jackknife de $1^{\mathrm{a}}$ ordem, jackknife de $2^{\mathrm{a}}$ ordem, bootstrap, Michaelis-Menten, utilizando-se 50 casualizações com abundância de classes igual a 10, foram feitos pelo programa EstimateS Richness Estimator Versão 6.0b1 (Colwell 2000).

\section{RESULTADOS}

Riqueza de espécies. Nas cinco áreas de Vila Velha foram capturados 10.822 exemplares pertencentes a 1.659 espécies de 64 famílias (Tabela I). A maior riqueza de espécies foi observada na área fase 1, com 685 espécies, seguida da área fase 2, com 658; a área com menor número de espécies foi a fase 3 (517). As áreas em estágio sucessional menos avançado foram mais ricas em espécies que as mais conservadas. A área de povoamento de araucária, com forte penetração de 
Tabela I. Número de espécies por famílias de Coleoptera capturadas com malaise em cinco áreas do Parque Estadual de Vila Velha, Ponta Grossa, Paraná, de setembro de 1999 a agosto de 2000.

\begin{tabular}{|c|c|c|c|c|c|c|}
\hline Família & Borda & Araucária & Fase 1 & Fase 2 & Fase 3 & Total \\
\hline Anobiidae & 2 & 3 & 5 & 8 & 8 & 16 \\
\hline Anthicidae & 0 & 0 & 1 & 1 & 0 & 2 \\
\hline Anthribidae & 9 & 8 & 9 & 6 & 7 & 21 \\
\hline Apionidae & 0 & 0 & 0 & 0 & 1 & 1 \\
\hline Attelabidae & 0 & 1 & 2 & 4 & 0 & 6 \\
\hline Belidae & 0 & 1 & 1 & 0 & 0 & 2 \\
\hline Bostrichidae & 0 & 0 & 0 & 1 & 1 & 2 \\
\hline Brentidae & 8 & 4 & 9 & 7 & 3 & 23 \\
\hline Buprestidae & 10 & 8 & 9 & 6 & 4 & 26 \\
\hline Cantharidae & 10 & 3 & 7 & 9 & 0 & 20 \\
\hline Carabidae & 24 & 4 & 11 & 13 & 12 & 41 \\
\hline Cerambycidae & 51 & 39 & 74 & 47 & 28 & 128 \\
\hline Ceratocanthidae & 0 & 0 & 0 & 0 & 1 & 1 \\
\hline Chelonariidae & 1 & 1 & 1 & 3 & 2 & 3 \\
\hline Chrysomelidae & 91 & 57 & 86 & 77 & 64 & 233 \\
\hline Clambidae & 0 & 0 & 2 & 0 & 1 & 2 \\
\hline Cleridae & 10 & 9 & 18 & 7 & 4 & 24 \\
\hline Coccinellidae & 32 & 19 & 13 & 12 & 18 & 53 \\
\hline Colydiidae & 3 & 2 & 0 & 0 & 0 & 4 \\
\hline Corylophidae & 3 & 3 & 4 & 0 & 1 & 6 \\
\hline Cucujidae & 4 & 2 & 5 & 1 & 2 & 8 \\
\hline Curculionidae & 89 & 66 & 93 & 75 & 36 & 263 \\
\hline Dermestidae & 1 & 1 & 2 & 0 & 1 & 3 \\
\hline Dytiscidae & 0 & 1 & 1 & 1 & 0 & 1 \\
\hline Elateridae & 32 & 27 & 38 & 52 & 38 & 84 \\
\hline Endomychidae & 4 & 7 & 6 & 4 & 9 & 16 \\
\hline Erotylidae & 7 & 9 & 9 & 18 & 11 & 41 \\
\hline Eucinetidae & 1 & 0 & 1 & 0 & 1 & 1 \\
\hline Eucnemidae & 5 & 9 & 14 & 12 & 14 & 23 \\
\hline Geotrupidae & 1 & 2 & 3 & 4 & 2 & 7 \\
\hline Histeridae & 0 & 1 & 0 & 4 & 3 & 6 \\
\hline Hydrophilidae & 0 & 0 & 0 & 2 & 1 & 2 \\
\hline Lampyridae & 11 & 15 & 11 & 11 & 8 & 28 \\
\hline Languriidae & 1 & 1 & 0 & 0 & 2 & 4 \\
\hline Lathridiidae & 3 & 0 & 1 & 2 & 1 & 3 \\
\hline Leiodidae & 0 & 1 & 1 & 5 & 5 & 7 \\
\hline Lucanidae & 1 & 0 & 0 & 0 & 0 & 1 \\
\hline Lycidae & 4 & 8 & 9 & 7 & 4 & 11 \\
\hline Megalopodidae & 1 & 5 & 4 & 5 & 4 & 9 \\
\hline Melandryidae & 3 & 3 & 2 & 2 & 2 & 5 \\
\hline Melyridae & 2 & 0 & 1 & 0 & 0 & 2 \\
\hline Monommatidae & 0 & 3 & 2 & 0 & 0 & 4 \\
\hline Mordelidae & 27 & 59 & 62 & 68 & 59 & 113 \\
\hline Mycetophagidae & 1 & 3 & 3 & 2 & 2 & 5 \\
\hline Mycteridae & 1 & 0 & 0 & 1 & 1 & 1 \\
\hline Nemonychidae & 1 & 1 & 0 & 0 & 0 & 1 \\
\hline Nitidulidae & 5 & 18 & 19 & 22 & 15 & 48 \\
\hline Oedemeridae & 4 & 4 & 1 & 3 & 3 & 8 \\
\hline Phalacridae & 1 & 3 & 3 & 1 & 3 & 6 \\
\hline Phengodidae & 2 & 3 & 2 & 1 & 1 & 3 \\
\hline Platypodidae & 2 & 1 & 10 & 3 & 7 & 12 \\
\hline Ptiliidae & 0 & 1 & 0 & 0 & 0 & 1 \\
\hline Ptilodactylidae & 8 & 14 & 13 & 11 & 5 & 17 \\
\hline Rhipiphoridae & 2 & 0 & 1 & 1 & 0 & 4 \\
\hline Scarabaeidae & 18 & 23 & 35 & 38 & 18 & 68 \\
\hline Scirtidae & 0 & 0 & 1 & 0 & 1 & 2 \\
\hline Scolytidae & 7 & 14 & 19 & 8 & 13 & 34 \\
\hline Scraptiidae & 0 & 2 & 0 & 1 & 1 & 3 \\
\hline Scydmaenidae & 0 & 2 & 1 & 0 & 0 & 2 \\
\hline Silvanidae & 4 & 2 & 1 & 1 & 2 & 6 \\
\hline Staphylinidae & 36 & 46 & 40 & 61 & 66 & 125 \\
\hline Tenebrionidae & 8 & 14 & 15 & 28 & 19 & 50 \\
\hline Throscidae & 0 & 1 & 0 & 0 & 0 & 1 \\
\hline Trogositidae & 2 & 2 & 3 & 2 & 3 & 6 \\
\hline Total de Espécies & 553 & 536 & 684 & 658 & 518 & 1659 \\
\hline
\end{tabular}

elementos herbáceos, arbustivos e arbóreos da mata natural, apresentou uma riqueza de espécies próxima à da área mais conservada (fase 3). A área de borda teve uma riqueza de espécies menor que aquelas das áreas em estágios menos avançados de sucessão (fase 1 e 2).

Curva de acumulação de espécies. O gráfico de acumulação de espécies capturadas a cada semana, em cada área (Fig. 1), mostra que nos primeiros três meses de coleta (13 semanas, de setembro a novembro) os percentuais variaram de cerca de 44\% (área de araucária) a 57\% (área fase 1) do total de espécies capturadas na respectiva área; e, até o fim dos primeiros 5 meses (22 semanas, de setembro a janeiro), continuou o acentuado acréscimo de novas espécies, resultando numa pronunciada linha ascendente. Neste período foram capturados cerca de $77 \%$ na área de araucária até $86 \%$ na área fase $1 . \mathrm{O}$ acréscimo de novas espécies chegou próximo à estabilidade em fins de maio, na $39^{\text {a }}$ semana, com cerca de $90 \%$ das espécies nas áreas de borda e araucária, e 97\% na área fase 1. Apesar das diferenças, as curvas de acumulação são significativamente correlacionadas (prob. $<0,01$ ).

Estimativa de riqueza. Utilizando os dados semanais de captura obteve-se, através de vários métodos (Chao1, Chao2, jackknife1, jackknife2, bootstrap, Michaelis-Menten), a estimativa da riqueza para cada área (Tabela II).

Por todos os estimadores, a área fase 1, em estágio inicial/ intermediário de sucessão vegetal, deve ser a mais rica em espécies, repetindo a maior riqueza definida pelo número de espécies efetivamente capturadas. Por outro lado, a área fase 3, a mais conservada, ou em estágio mais avançado de sucessão, foi a que teve os menores valores estimados de riqueza, não só dentre as áreas em sucessão, mas também com relação à área de borda e à de araucária. Numa leitura dos valores percentuais observa-se uma tendência dos diferentes métodos estimarem a riqueza de espécies em números proporcionalmente menores das áreas menos conservadas para as mais conservadas.

Espécies raras. Entre todas as áreas houve uma relativa homogeneidade quanto à presença de "singletons" locais, "singletons" únicas e "doubletons" (Colwell 2000; Novotný \& Basset 2000), variando pouco percentualmente (Tabela III). $\mathrm{O}$ percentual de espécies únicas (aquelas que ocorreram em apenas uma amostra, independente do número de indivíduos) também mostrou uma relativa semelhança entre as áreas, entre 21 a 33\%. Todas as classes de espécies raras, considerando também as espécies únicas (Coddington et al. 1996), foram percentualmente mais numerosas nas áreas mais agredidas antropicamente, declinando da área de borda para a área fase 3.

Índices de diversidade e uniformidade. $\mathrm{O}$ cálculo dos índices de diversidade de Brillouin (HB) e de Shannon (H') apresentaram resultados muito semelhantes, não apenas quanto aos valores absolutos, como também na ordem hierarquizada dos valores de diversidade das áreas (Tabela IV).

Assim, a área fase 1 foi a que se mostrou como a de maior diversidade; enquanto as áreas de borda e de araucária foram 


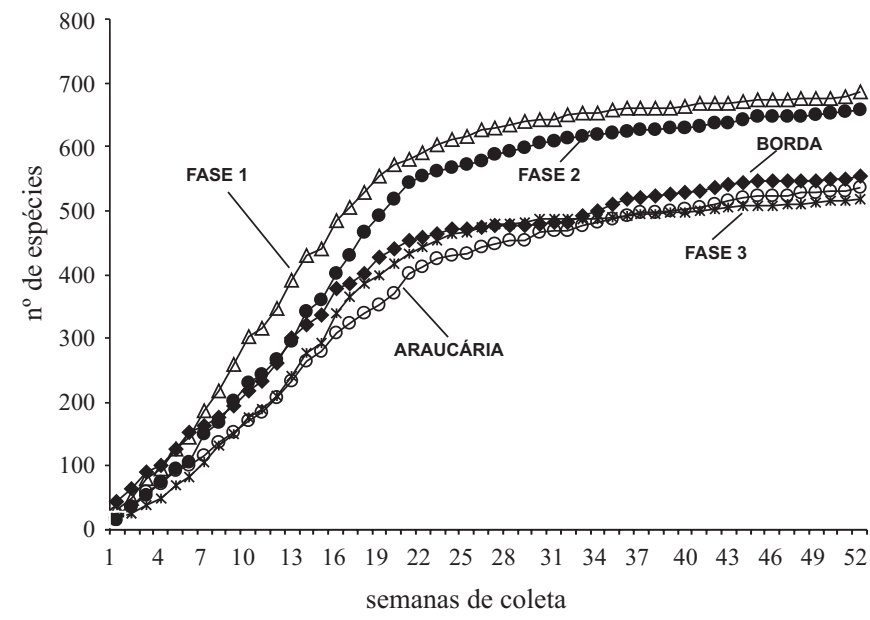

Fig. 1. Número acumulado de espécies de Coleoptera capturadas semanalmente por armadilha malaise em cinco áreas do Parque Estadual de Vila Velha, Ponta Grossa, Paraná, de setembro de 1999 a agosto de 2000 .

as de menor diversidade. Quanto à uniformidade, os índices não foram coerentes. Enquanto o de Berger \& Parker indicou a área fase 2 como sendo a mais uniforme, o índice de Shannon indicou a área fase 3 como mais uniforme, e a área fase 2 como aquela de menor uniformidade dentre todas as áreas.

Constância taxonômica das famílias com maior riqueza de espécies. Em cada área, apenas seis a sete famílias mais ricas representam cerca de $60 \%$ da riqueza de espécies. No conjunto das cinco áreas, em que foram arroladas 64 famílias, apenas nove foram responsáveis por cerca de $60 \%$ da riqueza total de espécies (Tabela V).

Curculionidae foi a família mais rica em espécies (263), seguida de Chrysomelidae (233), Cerambycidae (128), Staphylinidae (125), Mordelidae (113), Elateridae (84), Scarabaeidae (68), Coccinellidae (53) e Tenebrionidae (50). Nas áreas interiores (araucária e fases 1, 2 e 3), as seis primeiras famílias citadas foram sempre as mais ricas, apenas alternando a posição de maior riqueza. Na área de borda, a família Mordelidae foi substituída por Coccinellidae.

Em todas as áreas houve uma maior riqueza de espécies das famílias com hábitos herbívoros, exceto na área mais conservada (fase 3). Nesta, a família Staphylinidae, de hábito predominantemente carnívoro, foi a mais rica. Nesta mesma área, os Elateridae, também em sua maioria carnívoros, tiveram proporcionalmente uma alta riqueza.

\section{DISCUSSÃO}

Riqueza de espécies. As florestas secundárias mostram uma produtividade superior à das florestas primárias; isto pode ser explicado pelas condições relativamente estáticas de uma floresta primária, enquanto que as florestas secundárias sucessionais apresentam uma heterogeneidade estrutural mais alta, uma maior dinâmica e uma maior produtividade (Brown \& Lugo 1990; Wagner 2000). Devido à maior penetração de luz no sub-bosque das florestas secundárias em sucessão, em razão de uma maior abertura do dossel, há uma densidade maior de folhas e um aumento na produção de folhas jovens (Aide \& Zimmermann 1990; Aide 1992). Southwood et al. 1979 e Brown \& Hyman 1986 observaram que a diversidade de plantas e insetos herbívoros era maior nos estágios iniciais de sucessão vegetal, decaindo continuamente para os estágios mais avançados, principalmente no sub-bosque. Em Vila Velha, a maior riqueza de espécies de Coleoptera nas áreas florestais secundárias em estágio inicial a intermediário de sucessão vegetal também deve ser produto das condições acima indicadas, corroborando o observado por Southwood et al. 1979 e Brown \& Hyman 1986. A maior riqueza de espécies de Coleoptera em áreas menos conservadas também foi observada por Lawton et al. (1998), em estudo da fauna capturada por malaise, na Reserva Florestal Mbalmayo, em Camarões.

Os dados relativos ao número de espécies das áreas de araucária e da fase 3 são os mais baixos dentre todas as áreas, repetindo o observado nos estudos com a abundância de indivíduos por famílias (Ganho \& Marinoni 2003). Se a menor riqueza de espécie da área fase 3 pode ser explicada pela sua condição de floresta secundária em estágio avançado de sucessão, a menor riqueza da área de araucária possivelmente é devida à sua origem. Constituindo-se em uma capoeira artificial de araucária, não teve uma evolução natural dos estágios sucessionais em razão do porte das árvores que dificulta a entrada de luz, impedindo o desenvolvimento dos estratos inferiores, resultando na baixa diversidade de espécies de plantas (F.Galvão \& Y.S.Kuniyoshi in Ganho \& Marinoni 2003). Brown \& Lugo (1990) constataram que as florestas secundárias derivadas de uma área abandonada após práticas

Tabela II. Número de espécies de Coleoptera capturadas por malaise e as estimativas de riqueza de espécies por diferentes métodos em cinco áreas do Parque Estadual de Vila Velha, Ponta Grossa, Paraná, de setembro de 1999 a agosto de 2000 (número entre parênteses indica porcentagem de espécies passíveis de existirem a mais que as já capturadas em cada área).

\begin{tabular}{lccccc}
\hline & Borda & Araucária & Fase 1 & Fase 2 & Fase 3 \\
\hline Espécies capturadas & 553 & 536 & 684 & 658 & 18 \\
Chao1 & $1156 \pm 95(109)$ & $982 \pm 73(83)$ & $1316 \pm 93(92)$ & $1271 \pm 95(93)$ & $937 \pm 73(81)$ \\
Chao2 & $1236 \pm 108(123)$ & $1050 \pm 83(96)$ & $1382 \pm 102(102)$ & $1272 \pm 92(93)$ & $968 \pm 77(87)$ \\
Jackknife1 & $896 \pm 50(62)$ & $843 \pm 35(57)$ & $1069 \pm 54(56)$ & $1014 \pm 44(54)$ & $790 \pm 39(53)$ \\
Jackknife2 & $1148 \pm 17(107)$ & $1055 \pm 10(97)$ & $1344 \pm 15(96)$ & $1263 \pm 13(92)$ & $978 \pm 10(89)$ \\
Michaelis-Menten & $1127(103)$ & $975(82)$ & $1122(64)$ & $956(45)$ & $812(57)$ \\
Bootstrap & $695 \pm 10(25)$ & $665 \pm 7(24)$ & $845 \pm 9(23)$ & $807 \pm 8(23)$ & $632 \pm 6(22)$ \\
\hline
\end{tabular}


Tabela III. Número total de espécies, número de "singletons" únicas e "singletons" locais, "doubletons” e espécies únicas de Coleoptera, e seus percentuais, capturadas com malaise, em cinco áreas do Parque Estadual de Vila Velha, Ponta Grossa, Paraná, de setembro de 1999 a agosto de 2000 .

\begin{tabular}{|c|c|c|c|c|c|}
\hline Espécies /área & Borda & Araucária & Fase 1 & Fase 2 & Fase 3 \\
\hline Total & 553 & 536 & 684 & 658 & 518 \\
\hline "Singletons" únicas & $181(33 \%)$ & $130(24 \%)$ & $173(25 \%)$ & $167(25 \%)$ & $110(21 \%)$ \\
\hline "Singletons" locais & $335(60 \%)$ & $296(55 \%)$ & $378(55 \%)$ & $347(53 \%)$ & $266(51 \%)$ \\
\hline "Doubletons" & $92(17 \%)$ & $97(18 \%)$ & $112(16 \%)$ & $97(15 \%)$ & $83(16 \%)$ \\
\hline Únicas & $349(63 \%)$ & $313(58 \%)$ & $392(57 \%)$ & $363(55 \%)$ & $279(54 \%)$ \\
\hline
\end{tabular}

agrícolas continha maior número de espécies de plantas do que uma área de plantação de café abandonada, onde também se encontravam árvores sombreadoras dos cafeeiros e ainda frutíferas. Apesar das diferentes origens das florestas secundárias da área de araucária e da fase 3 , as características estruturais assemelhadas, que são definidas pela presença de um estrato médio (não encontrado nas outras áreas), podem também ter sido importantes para estabelecer a menor riqueza de espécies, no estrato do sub-bosque.

A área de borda, que se caracteriza como um ecótono, teve uma riqueza de espécies inferior às áreas em estágio inicial a intermediário de sucessão; foi, no entanto, superior à riqueza da área mais conservada, bem como da área de araucária, apesar de ter um menor número de indivíduos e de famílias que estas (Ganho \& Marinoni 2003).

Considerando cada uma das áreas de Vila Velha como sendo representativas de comunidades diferentes de besouros, com número de espécies que variou de 517 a 685 , vê-se que estes valores são muito expressivos quando comparados aos dados apresentados por Dajoz (2001). Este autor, fazendo uma generalização, relacionou uma média de 190 espécies para comunidades de florestas temperadas e de 784 espécies para florestas tropicais. As florestas temperadas situam-se em vastas áreas geográficas e apresentam um largo espectro climático, variando das mais baixas às mais altas latitudes e, numa mesma latitude, sendo afetada pela altitude. A menor riqueza de espécies para as zonas temperadas, referida por Dajoz (op.cit.), deve ser produto de levantamentos em áreas mais frias, de mais altas latitude e altitude que as de Vila Velha. Nesta localidade, considerada também de clima temperado, não há a ocorrência de neve como nas áreas de mais alta latitude e altitude. Os dados de diversidade $\alpha$ (alfa) apontam para altos valores em Vila Velha, comparados a outros locais.

Acumulação de espécies. Apesar das diferenças observadas nas curvas de acumulação de espécies (Fig. 1) com base nos dados de cada uma das cinco áreas, houve alta correlação. Os percentuais do número acumulado de espécies observadas no fim dos três primeiros meses (setembro a novembro) de coleta nas cinco áreas variaram de 44 a $57 \%$ do total do ano, porém os percentuais anotados por Marinoni \& Dutra (1996), Marinoni et al. (1997) e Marinoni et al. (1999) nos levantamentos de Ctenuchidae, Saturniidae e Sphingidae (Lepidoptera), respectivamente, diferiram significativamente. Nestes levantamentos, nos três primeiros meses (agosto a outubro) de captura, já haviam sido observados 66,65 e 71\% das espécies. A possível causa desta diferença deve estar nos hábitos alimentares dos insetos envolvidos e no nível taxonômico usado como referência (ordem e família). Enquanto as famílias de Lepidoptera citadas são essencialmente filófagas (herbívoras) na fase larval, sendo mais abundantes quando há a emergência de folhas novas e, portanto, dependentes da fenologia das plantas, os Coleoptera apresentam, além do hábito filófago, todos os demais hábitos dos herbívoros, e mais os hábitos fungívoro, detritívoro e carnívoro. E as famílias que apresentam estes últimos hábitos teriam, como os Staphylinidae e Elateridae, seus picos de abundância, e de maior riqueza de espécies, retardados em relação aos das famílias de espécies herbívoras mais abundantes (Curculionidae, Chrysomelidae, Cerambycidae, Mordelidae). Desta forma, a maior atividade de famílias carnívoras, posterior à das famílias herbívoras, resultou num período mais longo de acréscimo de outras espécies à curva de acumulação de cada uma das áreas (setembro a janeiro), até chegar a uma quase estabilização em fins de maio.

Estimativa de riqueza. A adequação do emprego de cada um dos diferentes métodos de estimativa aos dados obtidos em diferentes levantamentos não é bem conhecida. Mas, em razão dos dados de Vila Velha terem apresentado mais de 50\% de "singletons" locais, o melhor estimador deveria ser Chao 1 (Chao 1984 apud Coddington et al. 1996), que indicou altos valores de riqueza para todas as áreas, juntamente com o Chao 2 e jackknife2. Opostamente, o bootstrap que resultou nas mais baixas estimativas de riqueza de espécies em Vila Velha, com valores mais próximos da riqueza efetivamente observada, foi

Tabela IV. Diversidade e uniformidade das espécies de Coleoptera, capturadas com malaise em cinco áreas do Parque Estadual de Vila Velha, Ponta Grossa, Paraná, de setembro de 1999 a agosto de 2000. (N) número de indivíduos; (S) número de espécies; (HB) índice de diversidade de Brillouin; ( $\mathrm{H}^{\prime}$ ) índice de diversidade de Shannon; (UBP) índice de uniformidade de Berger e Parker; (H'E) índice de uniformidade de Shannon.

\begin{tabular}{lcccccc}
\hline & $\mathrm{N}$ & $\mathrm{S}$ & $\mathrm{HB}$ & $\mathrm{H}^{\prime}$ & $\mathrm{UBP}$ & $\mathrm{H}^{\prime} \mathrm{E}$ \\
\hline Borda & 1699 & 554 & 5,08 & 5,48 & 19,53 & 0,867 \\
Araucária & 1817 & 536 & 5,10 & 5,47 & 19,97 & 0,870 \\
Fase 1 & 2477 & 685 & 5,28 & 5,63 & 21,17 & 0,862 \\
Fase 2 & 2946 & 658 & 5,20 & 5,50 & 25,40 & 0,848 \\
Fase 3 & 1883 & 517 & 5,18 & 5,52 & 22,53 & 0,883 \\
\hline
\end{tabular}


Tabela V. Nove famílias de Coleoptera responsáveis por $60 \%$ da riqueza de espécies, capturadas com armadilha malaise em cinco áreas do Parque Estadual de Vila Velha, Ponta Grossa, Paraná, de setembro de 1999 a agosto de 2000. (S) Número de espécies; (\%) Porcentagem do total de espécies da área; (C) Classificação da família no conjunto das $64 \mathrm{em}$ cada uma das áreas. Números em negrito destacam a área onde a família apresentou o maior número de espécies. * O valor não foi incluído para obtenção dos cerca de $60 \%$.

\begin{tabular}{|c|c|c|c|c|c|c|c|c|c|c|c|c|c|c|c|c|c|c|}
\hline & \multicolumn{2}{|l|}{ Total } & \multicolumn{3}{|c|}{ Borda } & \multicolumn{3}{|c|}{ Araucária } & \multicolumn{3}{|c|}{ Fase 1} & \multicolumn{3}{|c|}{ Fase 2} & \multicolumn{3}{|c|}{ Fase 3} & \\
\hline & $\mathrm{S}$ & $\%$ & $\mathrm{C}$ & $\mathrm{S}$ & $\%$ & $\mathrm{C}$ & $\mathrm{S}$ & $\%$ & $\mathrm{C}$ & $\mathrm{S}$ & $\%$ & $\mathrm{C}$ & $\mathrm{S}$ & $\%$ & $\mathrm{C}$ & $\mathrm{S}$ & $\%$ & $\mathrm{C}$ \\
\hline Curculionidae & 263 & 15,9 & $1^{\mathrm{o}}$ & 89 & 16,1 & $2^{o}$ & 66 & 12,3 & $1^{\mathrm{o}}$ & 93 & 13,6 & $1^{\mathrm{o}}$ & 75 & 11,4 & $2^{o}$ & 36 & 7,0 & $5^{\circ}$ \\
\hline Chrysomelidae & 233 & 14,0 & $2^{\circ}$ & 91 & 16,4 & $1^{\mathrm{o}}$ & 57 & 10,6 & $3^{\circ}$ & 89 & 13,0 & $2^{o}$ & 77 & 11,7 & $1^{\mathrm{o}}$ & 64 & 12,4 & $2^{\circ}$ \\
\hline Cerambycidae & 128 & 7,7 & $3^{\circ}$ & 51 & 9,2 & $3^{\circ}$ & 39 & 7,3 & $5^{\circ}$ & 74 & 10,8 & $3^{\circ}$ & 47 & 7,1 & $6^{\circ}$ & 29 & 5,6 & $6^{\circ}$ \\
\hline Staphylinidae & 125 & 7,5 & $4^{\circ}$ & 37 & 6,7 & $4^{\mathrm{o}}$ & 45 & 8,4 & $4^{\mathrm{o}}$ & 40 & 5,8 & $5^{\circ}$ & 61 & 9,3 & $4^{\mathrm{o}}$ & 66 & 12,7 & $1^{\circ}$ \\
\hline Mordelidae & 113 & 6,8 & $5^{\circ}$ & $* 27$ & & & 59 & 11,0 & $2^{\circ}$ & 62 & 9,1 & $4^{\circ}$ & 68 & 10,3 & $3^{\circ}$ & 59 & 11,4 & $3^{\circ}$ \\
\hline Elateridae & 84 & 5,1 & $6^{\circ}$ & 32 & 5,8 & $5^{\circ}$ & 27 & 5,0 & $6^{\circ}$ & 38 & 5,5 & $6^{\circ}$ & 52 & 7,9 & $5^{\circ}$ & 37 & 7,2 & $4^{\circ}$ \\
\hline Scarabaeidae & 68 & 4,1 & $7^{\circ}$ & $* 18$ & & & 23 & 4,3 & $7^{\circ}$ & 35 & 5,1 & $7^{\circ}$ & $* 38$ & & & $* 17$ & & \\
\hline Coccinellidae & $* 53$ & & $8^{\circ}$ & 32 & 5,8 & $6^{\circ}$ & *19 & & & $* 13$ & & & $* 12$ & & & $* 18$ & & \\
\hline Tenebrionidae & $* 50$ & & $9^{\circ}$ & $* 8$ & & & $* 14$ & & & $* 15$ & & & $* 26$ & & & 19 & 3,7 & $7^{\circ}$ \\
\hline Total & & 61,1 & & & 60,0 & & & 58,9 & & & 62,9 & & & 57,7 & & & 60,0 & \\
\hline
\end{tabular}

reconhecido como bom indicador por Palmer (1990). Deve ser notado que nos estudos desse autor, os dados eram oriundos de coleta por quadrados, sem as possíveis implicações decorrentes do uso de pseudo-replicações de amostragem por malaise que, como no caso do presente trabalho, foram amostras por semana e por ano. Por outro lado, Brose (2002) fez um estudo dos estimadores, a partir da captura de Carabidae com armadilha de solo, e concluiu ser o estimador Chao2 o mais correto. Este estimador, que no presente estudo apresentou os mais altos valores de riqueza (na área de araucária e fase 3 levemente ultrapassada pelo jackknife 2), indicou haver ainda de 87 a $123 \%$ de espécies a serem capturadas, enquanto que pelo estimador bootstrap haveria apenas mais 22 a $25 \%$ de espécies, além das já observadas. Os valores percentuais indicam uma tendência da riqueza estimada declinar das áreas menos conservadas para as mais conservadas. Estes dados confirmam a influência da riqueza das espécies "singletons" e "doubletons" nos valores estimados de riqueza (Coddington et al. 1996), pois esta também é a tendência da riqueza das espécies raras nas diferentes áreas, como se observa na Tabela III.

$\mathrm{O}$ "ranking" dos estimadores com base nos valores encontrados foi muito semelhante ao observado por Carlton \& Robison (1998), em estudo com besouros do folhiço, no Arkansas, Estados Unidos. Ordenamento semelhante também pode ser visto em Brose (op.cit.). Esta igualdade de ordenamento dos estimadores já foi indicada por Coddington et al. (1996), tanto para dados de fauna tropical como temperada.

A dificuldade de reconhecer qualquer um dos métodos como sendo o que indicou a possível riqueza de cada uma das áreas de Vila Velha, remete a uma continuidade do estudo, com resultado possivelmente mais ajustado, que poderá ser produzido com o estudo do material levantado nas mesmas condições durante os anos de agosto de 2000 a julho de 2002.

Espécies raras. Mesmo considerando a possibilidade do efeito de massa (Shmida \& Wilson 1985), que poderia ocasionar a presença de um maior número de "singletons" e "doubletons" entre as áreas mais próximas, foi possível constatar que as áreas mais distantes espacialmente (áreas fase 2 e fase 3 ), apresentaram também grande número de espécies "singletons" e "doubletons". A proporção de espécies "singletons" locais foi alta, decrescendo das áreas mais alteradas pela ação antrópica para as menos alteradas. $\mathrm{O}$ mesmo fenômeno foi observado quanto às "singletons" únicas. As proporções registradas em Vila Velha (entre 21 e $33 \%$ ) foram muito próximas àquelas citadas por Novotný \& Basset (2000) ao estudarem as comunidades de insetos herbívoros em diferentes espécies de árvores na Nova Guiné. Estes autores consideraram que a proporção constante de espécies raras seria determinada pelos mesmos fatores, incluindo entre eles a produção e palatabilidade das folhas, e da densidade das árvores, para os insetos mastigadores, bem como da espessura das folhas para os sugadores. A proporção quase semelhante de espécies raras, mas concomitantemente crescente entre as áreas de Vila Velha, em razão dos diferentes níveis de antropização, também permite inferir sobre a existência de fatores comuns determinantes de tais riquezas de espécies. Mas, como neste levantamento são incluídas espécies de todos os grupos tróficos de Coleoptera, entre os fatores incluir-se-iam outros mais que apenas as características foliares para os besouros herbívoros. Os resultados semelhantes, alcançados em ambos os levantamentos, gerados a partir de metodologias diferentes, e envolvendo grupos taxonômicos e tróficos diferentes, faz vislumbrar a existência de fenômenos ecológicos comuns para a existência de espécies comuns e raras. Nas comunidades de Coleoptera de Vila Velha, as proporções diferenciadas, aumentando o número de espécies comuns e diminuindo o de espécies raras nas áreas mais conservadas, pode estar relacionada também à menor produtividade das florestas mais maduras. A menor riqueza de espécies raras em florestas maduras já foi referida por Hutcheson \& Jones (1999).

Índices de diversidade e uniformidade. Apesar dos índices serem menos informativos sobre as características do ambiente que a composição de espécies (Penev 1996; Scott \& Anderson 2003), os valores dos diferentes índices (de diversidade de Brillouin e de Shannon; de uniformidade de Berger \& Parker e 
de Shannon) foram indicativos de uma alta diversidade e de alta uniformidade em todas as áreas de Vila Velha. Porém, mais importante que os índices observados em cada uma das áreas, e das relações de maior ou menor diversidade/uniformidade de cada uma delas, foi a constatação dos altos valores resultantes, muito superiores aos citados em diferentes estudos de várias regiões, envolvendo a fauna de Coleoptera (Davies et al. 1997; Hutcheson \& Jones 1999).

A constância taxonômica das famílias com maior riqueza de espécies. Uma comparação entre os dados de abundância por família de Coleoptera (Marinoni \& Dutra 1997; Ganho \& Marinoni 2003), e os dados de riqueza de espécies por família, mostra que ao nível de $60 \%$ do total da abundância ou do total da riqueza de espécies, são arroladas as mesmas famílias. Assim, neste valor percentual houve uma constância taxonômica ao nível de família nos dois parâmetros: abundância e riqueza de espécies.

Caso esta constância seja observada em outros levantamentos, haverá possibilidade de que estudos concentrados na análise de apenas $60 \%$ de todo material coletado encontrem padrões ecológicos (tróficos e estruturais) e/ou de diversidade (riqueza de espécies e de abundância) que venham a definir os Coleoptera como importante indicador ecológico e de diversidade em áreas florestadas. A maior vantagem das análises, envolvendo apenas $60 \%$ do material coletado, seria o manejo dos exemplares nos processos de montagem e identificação. Neste último caso, o processo ficaria circunscrito às famílias mais comuns e mais fáceis de serem reconhecidas.

A maior representatividade de espécies de famílias herbívoras em áreas menos conservadas está de acordo com o observado em vários estudos (Morris 1980; Heliövaara \& Väisänen 1984; Hutcheson 1990; Marinoni \& Dutra 1997).

Não há na literatura dados de captura obtidos com armadilha malaise sobre riqueza de espécies por família para comparar com os dados deste trabalho. Os trabalhos de Hutcheson (1990) e de Hutcheson \& Jones (1999), também com armadilha malaise, não trazem informações sobre as famílias mais ricas. Em estudos sobre a fauna de Coleoptera de copa de árvores, com uso de fumigação, Davies et al. (1997) referem-se à existência de constância taxonômica em escala global, quando considerada a riqueza de espécies em cada família; fato também reconhecido por Wagner (2000). No entanto, Hammond et al. (1997), em um estudo na Indonésia (Sulawesi), no mesmo estrato florestal, empregando diferentes métodos de coleta (fumigação, malaise, armadilhas com isca, armadilha luminosa) reconheceram outras famílias como as mais ricas. De fato, a utilização de diferentes métodos de coleta resulta na captura de diferentes famílias, como já discutido por Marinoni \& Ganho (2003). Este fato evidencia a dificuldade de serem encontrados padrões quando são comparadas faunas de diferentes locais, coletadas por diferentes métodos.

\section{CONSIDERAÇÕES FINAIS}

As análises sobre a fauna de Coleoptera capturada por malaise, no Parque Estadual de Vila Velha, permitem destacar que:

- a diversidade $\alpha$ (alfa) é alta, enquanto a diversidade $\gamma$ (gama), preliminarmente considerada alta, requer dados comparativos para uma melhor avaliação;

- as áreas em estágio inicial a intermediário de sucessão vegetal apresentaram maior riqueza de espécies que as em estágio mais avançado;

- a maior riqueza de espécies nas áreas em sucessão menos avançada possivelmente é conseqüência da maior produtividade da vegetação e maior diversidade de plantas;

- as curvas de acumulação de espécies de Coleoptera têm as aproximações da assíntota retardadas, possivelmente devido aos diferentes hábitos alimentares do táxon, quando comparadas às curvas definidas a partir de grupos taxonômicos de hábitos herbívoros, fundamentalmente filófagos;

- os estimadores de riqueza de espécies apontam para a possibilidade de existirem ainda muitas espécies a serem capturadas, em um período maior de coleta;

- as proporções assemelhadas do número de espécies "singletons", "doubletons" e únicas, decrescendo das áreas menos conservadas para as mais conservadas, mostram elementos que podem configurar a existência de padrões de riqueza das espécies raras em função de níveis de conservação;

- se confirmada a existência de um padrão taxonômico envolvendo as famílias mais ricas em espécies e mais abundantes, ao nível de $60 \%$, os Coleoptera poderão ser utilizados como táxon indicador ecológico e de biodiversidade em áreas florestadas;

- houve predominância de famílias com hábitos carnívoros nas áreas mais conservadas, e de famílias com hábitos herbívoros nas demais áreas.

Agradecimentos. A todos os que colaboraram no projeto de levantamento da fauna entomológica no Parque Estadual de Vila Velha (PROVIVE) já relacionados Ganho \& Marinoni 2003 e Marinoni \& Ganho 2003 e a Cibele S. Ribeiro-Costa e Luciane Marinoni pela participação nas coletas de 2000 a 2002, e nas discussões envolvendo as faunas de Coleoptera e Diptera.

\section{REFERÊNCIAS}

Aide, T. M. 1992. Patterns of leaf development and herbivory in a tropical understory community. Ecology 74: 455-466.

Aide, T. M. \& J. K. Zimmerman. 1990. Patterns of insect herbivory, growth, and survivorship in juveniles of a neotropical liana. Ecology 71: $1412-1421$

Alatalo, Ra. \& Ri. Alatalo. 1977. Components of diversity: multivariate analysis with interaction. Ecology (Durham) 58: 900-906.

Brose, U. 2002. Estimating species richness of pitfall catches by nonparametric estimators. Pedobiologia 46: 101-107.

Brown, S. \& A. E. Lugo. 1990. Tropical secondary forests. Journal of Tropical Ecology 6: 1-32.

Brown, V. K. \& P. S. Hyman. 1986. Successional communities of plants and phytophagous Coleoptera. Journal of Ecology 74: 963-975.

Bullock, J. A. 1971. The investigation of samples containing many species. I. Sample description. Biological Journal of Linnean Society 3: 1-21. 
Carlton, C. E. \& H. W. Robinson. 1998. Diversity of litter-dwelling beetles in the Ouachita highlands of Arkansas, USA (Insecta: Coleoptera). Biodiversity and Conservation 7: 1589-1605.

Coddington, J. A; L. H. Young \& F. A. Coyle. 1996. Estimating spider species richness in a southern Appalachian cove hardwood forest. The Journal of Arachnology 24: 111-128.

Colwell, R. K. 2000. Estimates: Statistical estimation of species richness and shared species from samples. Version $6.0 \mathrm{~b} 1$. User's Guide Draft 7 March 2001. Disponível em: http:// viceroy.eeb.uconn.edu/estimates . Acesso em 20/03/2002.

Colwell, R. K. \& J. A. Coddington. 1994. Estimating terrestrial biodiversity through extrapolation. Philosophical Transactions of the Royal Society of London B 345: 101-118.

Dajoz, R. 2001. Composition spécifique et structure d'un peuplement de Coléoptères forestiers de l'Arizona (États-Unis). Comparaison avec des régions tempérées et tropicales. Bulletin de la Société Entomologique de France 106: 391-400.

Davies, J. G.; N. E. Sork; M. J. D. Brendell \& S. J. Hine. 1997. Beetle species diversity and faunal similarity in Venezuelan rainforest tree canopies, p. 85-103. In: Stork, N. E.; J. Adis \& R. K. Didham (Edits). Canopy Arthropods. London, Chapman \& Hall.

Ganho, N. G. \& R. C. Marinoni. 2003. Fauna de Coleoptera no Parque Estadual de Vila Velha, Ponta Grossa, Paraná, Brasil. Abundância e riqueza das famílias capturadas através de armadilhas malaise. Revista Brasileira de Zoologia 20: 727-236.

Gotelli, N. J. \& R. K. Colwell. 2001. Quantifying biodiversity: procedures and pitfalls in measurement and comparison of species richness. Ecology Letters 4: 379-391.

Hammond, P. M.; N. E. Stork \& M. J. D. Brendell. 1997. Tree-crown Beetles in context: a comparison of canopy and other ecotone assemblages in a lowland tropical forest in Sulawesi, p.184-223. In: Stork, N. E.; J. Adis \& R. K. Didham (Edits). Canopy Arthropods. London, Chapman \& Hall.

Harrison, S; S. J. Ross \& J. H. Lawton. 1992. Beta diversity on geographic gradients in Britain. Journal of Animal Ecology 61: $151-158$.

Heliövaara, K. \& R. Väisänen. 1984. Effects of modern forestry on northwestern European forest invertebrates: a synthesis. Acta Forestalia Fennica 189: 1-29.

Humphrey, J. W.; C. Hawes; A. J. Peace; R. Ferris-Kaan \& M. R. Jukes. 1999. Relationships between insect diversity and habitat characteristics in plantation forests. Forest Ecology and Management 113: 11-21.

Hutcheson, J. \& D. Jones. 1999. Spatial variability of insect communities in a homogenous system: measuring biodiversity using Malaise trapped beetles in a Pinus radiata plantation in New Zealand. Forest Ecology and Management 118: 93-105.

Hutcheson, J. 1990. Characterization of terrestrial insect communities using quantified, Malaise-trapped Coleoptera. Ecological Entomology 15: 143-151.

IBGE (Instituto Brasileiro de Geografia e Estatística). 1992. Manual técnico da vegetação brasileira. Rio de Janeiro, IBGE, Séries Manuais Técnicos em Geociências 1, 92p.

Koleff, P.; K. J. Gaston; J. J. Lennon. 2003. Measuring beta diversity for presence-absence data. Journal of Animal Ecology 72: $367-$ 382 .

Lawton, J. H. 1983. Plant architecture and the diversity of phytophagous insects. Annual Review Entomology 28: 23-39.

Lawton, J. H.; D. E. Bignell; B. Bolton; G. F. Bloemers; P. Eggleton; P. M. Hammond; M. Hodda; R. D. Holt; D. S. Srivastava \& A. D. Watt. 1998. Biodiversity inventories, indicator taxa and effects of habitat modification in tropical forest. Nature 39: 72-76.

Maack, R. 1981. Geografia Física do Estado do Paraná. Rio de Janeiro, José Olympio Editora, $450 \mathrm{p}$.

Magurran, A. E. 1988. Ecological diversity and its measurement. New Jersey, Princeton University Press, 179p.
Magurran, A. E. 2004. Measuring Biological Diversity. Oxford, Blackwell Science Ltd., 256p.

Marinoni, R. C. \& N. G. Ganho. 2003. A fauna de Coleoptera em áreas com diferentes condições florísticas no Parque Estadual de Vila Velha, Ponta Grossa, Paraná, Brasil. Abundância e riqueza das famílias capturadas através de armadilhas de solo. Revista Brasileira de Zoologia 20: 737-744.

Marinoni, R. C. \& R. R. C. Dutra. 1996. Levantamento da fauna entomológica no Estado do Paraná. II. Ctenuchidae (Lepidoptera). Revista Brasileira de Zoologia 13: 435-461.

Marinoni, R. C. \& R. R. C. Dutra. 1997. Famílias de Coleoptera capturadas com armadilha malaise em oito localidades do Estado do Paraná, Brasil. Diversidades alfa e beta. Revista Brasileira de Zoologia 14: 751-770.

Marinoni, R. C.; R. R. C. Dutra \& M. M. Casagrande. 1997. Levantamento da fauna entomológica no Estado do Paraná. III Saturniidae (Lepidoptera). Revista Brasileira de Zoologia 14: 473-495.

Marinoni, R. C.; R. R. C. Dutra \& O. H. H. Mielke. 1999. Levantamento da fauna entomológica no Estado do Paraná. IV. Sphingidae (Lepidoptera). Diversidade alfa e estrutura de comunidade. Revista Brasileira de Zoologia 16: 223-240.

McAleece, N. 1998. Bio Diversity Professional Beta. Disponível em: www.nrmc.demon.co.uk - Acesso em: 20/03/2002.

Metzger, J. P. 2001. O que é ecologia de paisagens? Biota Neotropica 1:1-9. Disponível em http://www.biotaneotropica.org.br

Moreno, C. E. \& G. Halffter. 2001. Spatial and temporal analysis of a, $\mathrm{b}$ and $\mathrm{g}$ diversities of bats in a fragmented landscape. Biodiversity and Conservation 10: $367-382$.

Morris, M. G. 1980. Insects and the environment in the United Kingdom. Atti XII Congresso Nazionale Italiano di Entomologia, Roma. p. 203-235.

Novotný, V \& Y. Basset. 2000. Rare species in communities of tropical insect herbivores: pondering the mystery of singletons. Oikos 89 : $564-572$.

Oliveira, E. B.; R. D. Miyazaki \& W. Severi. 1998. Cálculo de fatorial e seu uso no índice de Brillouin. Revista Brasileira de Biologia 58: $37-341$.

Palmer, M. W. 1990. The estimation of species richness by extrapolation. Ecology 71: 1195-1198.

Penev, L. 1996. Large-scale variation in carabid assemblages, with special reference to the local fauna concept. Annales Zoologici Fennici 33: 49-63.

Samways, M. J. 1995. Insect Conservation Biology. London, Chapman \& Hall, 358p.

Scott, W. A. \& R. Anderson. 2003. Temporal and spatial variation in carabid assemblages from the United Kingdom Environmental Change Network. Biological Conservation 110: 197-210.

Shmida, A. \& M. R. Wilson. 1985. Biological determinants of species diversity. Journal of Biogeography 12: 1-20.

Southwood, T. R. E.; V. K. Brown \& P. M. Reader. 1979. The relationships of plant and insect diversities in succession. Biological Journal of the Linnean Society 12: 327-348.

Townes, H. 1972. A light-weight Malaise trap. Entomological News 83: 239-247.

Veloso, H. P. \& L. Góes filho. 1982. Fitogeografia Brasileira. Classificação fisionômico-ecológica da vegetação neotropical. Boletim Técnico Projeto RADAMBRASIL. Série Vegetação. Salvador. $85 \mathrm{p}$.

Wagner, T. 2000. Influence of forest type and tree species on canopydwelling beetles in Budongo Forest, Uganda. Biotropica 32: 502514.

Whittaker, R. H. 1960. Vegetation of Siskiyou Mountains, Oregon and California. Ecological Monographs 22: 1-44.

Whittaker, R. H. 1972. Evolution and measurement of species diversity. Taxon 21: 213-251. 